\title{
Adequate Soliton Solutions to the Space-Time Fractional Telegraph Equation and Modified Third- Order KdV Equation through A Reliable Technique
}

\section{Ummay Sadia}

Jashore University of Science and Technology

Mohammad Asif Arefin

Jashore University of Science and Technology

Mustafa Inc

Firat University: Firat Universitesi

M. Hafiz Uddin ( $\square$ mh.uddin@just.edu.bd)

Jashore University of Science and Technology https://orcid.org/0000-0003-3725-5472

\section{Research Article}

Keywords: The space time-fractional Telegraph equation, the space time-fractional modified third-order $\mathrm{KdV}$ equation, conformable fractional derivative, traveling wave solution, the extended tanh-function method.

Posted Date: November 2nd, 2021

DOI: https://doi.org/10.21203/rs.3.rs-996854/v1

License: (c) (i) This work is licensed under a Creative Commons Attribution 4.0 International License. Read Full License 


\title{
Adequate Soliton Solutions to the Space-Time Fractional Telegraph Equation and Modified Third-Order KdV Equation through A Reliable Technique
}

\author{
Ummay Sadia ${ }^{1}$, Mohammad Asif Arefin ${ }^{1}$, Mustafa Inc ${ }^{2,3,4}$ M. Hafiz Uddin ${ }^{1, *}$ \\ ${ }^{1}$ Department of Mathematics, Jashore University of Science and Technology, Jashore-7408, \\ Bangladesh \\ ${ }^{2}$ Department of Computer Engineering, Biruni University, Istanbul, Turkey \\ ${ }^{3}$ Department of Mathematics, Science Faculty, Firat University, 23119 Elazig, Turkey \\ ${ }^{4}$ Department of Medical Research, China Medical University Hospital, China Medical \\ University, Taichung, Taiwan
}

"Corresponding Author: M. Hafiz Uddin (mh.uddin@just.edu.bd)

\begin{abstract}
The space-time fractional telegraph equation and the space-time fractional modified third-order Kdv equations are significant molding equations in theoretic physics, mathematical physics, plasma physics also other fields of nonlinear sciences. The space time-fractional telegraph equation, which appears in the investigation of an electrical communication line and includes voltage in addition to current which is dependent on distance and time, is also applied to communication lines of wholly frequencies, together with direct current, as well as highfrequency conductors, audio frequency (such as telephone lines), and low frequency (for example cable television) used in the extension of pressure waves into the lessons of pulsatory blood movement among arteries also the one-dimensional haphazard movement of bugs towards an obstacle. The presence of chain rule and the derivative of composite functions allows the nonlinear fractional differential equations (NLFDEs) to translate into the ordinary differential equation employing wave alteration. To explore such categories of resolutions, the extended tanh-method is accomplished via Conformable fractional derivatives. A power sequence in tanh was originally used as an ansatz to provide analytical solutions of the traveling wave type of certain nonlinear evolution equations. To convert this problem to a standard
\end{abstract}


differential equation, a partial complex transformation that has been summarized succinctly is utilized correctly thus, with all of the free parameters, numerous exact logical arrangements are required. The results are found as hyperbolic and rational functions involving parameters, when specific values are supplied to the parameters solitary wave solutions are formed from traveling wave solutions. The outcomes achieved in this study are king type, single soliton, double soliton, multiple solitons, bell shape, and other sorts of forms and we demonstrated that these solutions were validated through the Maple software. The proposed approach for solving nonlinear fractional partial differential equations has been developed to be operative, unpretentious, quick, and reliable to usage.

Keywords: The space time-fractional Telegraph equation; the space time-fractional modified third-order $\mathrm{KdV}$ equation; conformable fractional derivative; traveling wave solution; the extended tanh-function method.

MSC: 35C25, 35C07, 35C08, 35Q20, 76B25

\section{Introduction}

A huge scientific literature by numerous researchers from diverse fields of study has lately been accessible that agreement with dynamical classifications, engineering, in addition, mathematical physics well-defined by fractional differential equations. Ordinary differential equations are comprehensive to arbitrary (non-integer) order by fractional differential equations. Power law memory kernels capture nonlocal spatial besides temporal interactions in fractional differential equations. For the reason that fractional differential equations have so many dispensations in engineering as well as science, scientific learning on them has detonated. Several researchers [1-4] have looked at fractional differential equations and used innumerable explanations approaches. Fractional derivatives in addition to integrals were once 
regarded to be the domain of theoretic mathematics. However, many investigations in the preceding insufficient eras have recommended that fractional phenomenon is connected not only in mathematics but also in applied mathematics to engineering disciplines for example fluid mechanics, vascular mechanics, optical fibers, geochemistry, plasma physics, also to suchlike a great extent. The benefit of fractional derivatives in molding mechanical and electrical belongings of real materials turn into ostensible owing to the disregarding of properties in standard integer-order models. Fractional derivatives oblige as a foundation for telling the appearances of mathematics. Numerous outstanding approaches for resolving these schemes have been discovered in recent years in the utmost convenient publications on nonlinear FDEs for example the sub equation approach [5-7], the first integral approach [8,9], and the complex transform approach $[10,11]$, which propagate nonlinear fractional differential equations. FDEs well represent numerous key singularities in non-Brownian motion, signal processing, systems identification, control problems, viscoelastic materials, polymers, also supplementary areas of science [12]. Many authoritative approaches meant for gaining numerical and analytical results of FDEs have been established and developed, including the finite difference method [13], finite element method [14], Adomian decomposition method $[15,16]$, differential transform method [17], variational iteration method [18-20], homotopy perturbation method $[21,22]$, the double $\left(G^{\prime} / G, 1 / G\right)$-expansion method [23-26], the $\left(G^{\prime} / G^{2}\right)$ expansion method [27], and an efficient difference method [28]. The question of how to expand existing approaches to tackle other FDEs remains an intriguing and significant research topic. Numerous FDEs have been inspected and explained thanks to the efforts of many researchers, including the impulsive fractional differential equations[29][30], space-time fractional advection-dispersion equation [30-32], fractional generalized Burgers' fluid [33], and fractional heat and mass-transport equation [34], $\exp -(\varphi(\xi))$ expansion method [35], etc. 
The Telegraph equation appears in the learning of electrical signal circulation of pulsatory blood movement among arteries also a one-dimensional haphazard movement of bugs towards an obstacle. The telegraph equation has been proven to be a greater example because of narrating some fluid flow difficulties connecting interruptions when compared to the heat equation [36]. Several academics have solved the standard telegraph equation as well as the space or time-fractional telegraph equations. Biazar et al., [37] has been applied variational iteration method to attain an estimated explanation intended for the Telegraph equation. Yildirim [38] used homotopy perturbation method to gain systematic and estimated resolutions of the space time-fractional telegraph equations. Hassani, et al. [39] presents the transcendental Bernstein series (TBS) as a generalization of the classical Bernstein polynomials for solving the variable-order space time-fractional telegraph equation (V-STFTE). The space timefractional modified third-order $\mathrm{KdV}$ equation recite the circulation process of surface water waves. This equation seems within the electric circuit and multi constituent plasms and fluid mechanics, signal processing, hydrology, viscoelasticity and so on. Sohail, et al. [40] proposed method known as the $\mathrm{G}^{\prime} / \mathrm{G}$-expansion method and the fractional complex transform are successfully employed to obtain the exact solutions of fractional modified third-order $\mathrm{KdV}$ equations. Shah et al. [41] applied Adomian decomposition to display the efficiency of the technic used for together fractional and integer order the space of time-fractional modified third-order Kdv equation. Sepehrian and Shamohammadi [42] applied a radial basis function process for numerical resolution of time-fractional modified third-order $\mathrm{KdV}$ equation by radial basis functions and so many researchers using various types of method to acquire exact solution of space time-fractional modified third-order $\mathrm{KdV}$ equation.

The goal of this study is to use the extended tanh-function process to come up with innovative solutions to the above-mentioned equations. The extended tanh-function approach has yet to be used to explore the space-time fractional Telegraph and space-time fractional modified 
third-order Kdv equation. This technique has the advantage of allowing us to obtain more arbitrary constants and solutions types than other ways. In addition to the fundamental usage, it helps numerical solvers assess the accuracy of their conclusions and aids them with instability analysis.

The following is how the residual of the item is designed: In segment 2, we go through numerous definitions and characteristics of conformable fractional derivatives. Then show how to discover accurate traveling wave solutions to nonlinear fractional differential equations in segment 3. In segment 4, describe the new closed-form wave solution for the general spacetime fractional modified third-order $\mathrm{Kdv}$ equation and space-time fractional Telegraph equation. In segment 5, the findings and disputes are assessed through visual delegation and physical enlargement of the resolution, followed by a discussion of the conclusions

\section{Meaning and preamble}

Let, $f:[0, \infty) \rightarrow \mathbb{R}$, be a function. $f$ be $\alpha$-order "conformable derivative" is demarcated as [44]:

$K_{\alpha}(f)(t)=\lim _{\varepsilon \rightarrow 0} \frac{f\left(t+\varepsilon t^{1-\alpha}\right)-f(t)}{\varepsilon}$

For every $t>0, \alpha \in(0,1)$. If $f$ be $\alpha$-differentiable in nearly $(0, a), a>0$ in addition $\lim _{t \rightarrow 0^{+}} f^{(\alpha)}(t)$ be real, now $f^{(\alpha)}(0)=\lim _{t \rightarrow 0^{+}} f^{(\alpha)}(t)$. The theorems that survey high spot a limited axiom that are contented conformable derivatives.

Theorem 1: Suppose that $\alpha \in(0,1]$ and at a point $t>0 f, g$ be $\alpha$-differentiable. Hence

- $K_{\alpha}(x f+y g)=x K_{\alpha}(f)+y K_{\alpha}(g)$, for all $x, y \in \mathbb{R}$.

- $K_{\alpha}\left(t^{z}\right)=h t^{K-\alpha}$, for all $z \in \mathbb{R}$.

- $K_{\alpha}(u)=0$, for all constant function $f(t)=u$. 
- $K_{\alpha}(f g)=f K_{\alpha}(g)+g K_{\alpha}(f)$.

- $K_{\alpha}\left(\frac{f}{g}\right)=\frac{g K_{\alpha}(f)-f K_{\alpha}(g)}{g^{2}}$.

- Additionally, in case $f$ is differentiable, then $K T_{\alpha}(f)(t)=t^{1-\alpha} \frac{d f}{d t}$.

Some kinds of properties like as the chain law, Gronwall's inequality, integration procedures, the Laplace transform, Tailor series expansion, and the exponential function in terms of the conformable fractional derivative [43].

Theorem 2: In conformable differentiable, $f$ be a $\alpha$-differentiable function and also presume $g$ is also differentiable and described in assortment of $f$, so that

$M_{\alpha}(f \circ g)(t)=t^{1-\alpha} g^{\prime}(t) f_{g}(t)$

\section{Vital evidences in addition the enactment of the process}

The extended tanh function method for obtaining multiple exact solutions for nonlinear evolution equations (NLEEs) is described here which was summarized by Wazwaz [44]. To reveal the solution namely a polynomial in hyperbolic functions is the key idea behind the proposed methodology, and solve the variable coefficient PDE first solving the method which is including first-order ODEs also algebraic equations. To begin, we detain an NLEEs related with a function $U=U(x, t)$ as follows:

$R\left(u, D_{t}^{\alpha} u, D_{x}^{\beta} u, D_{t}^{\alpha} D_{t}^{\alpha} u, D_{t}^{\alpha} D_{x}^{\beta} u, D_{x}^{\beta} D_{x}^{\beta}, \ldots \ldots \ldots\right)=0,0<\alpha \leq 1,0<\beta \leq 1$,

where $u$ is an unidentified function with spatial and temporal derivatives $x$ also $t$, besides $R$ is a polynomial of $u(x, t)$ in addition its derivatives in which the maximum order of derivatives and nonlinear terms of the maximum order are interrelated. Let the conversion of waves.

$\xi=k \frac{x^{\beta}}{\beta}+c \frac{t^{\alpha}}{\alpha}, u(x, t)=u(\xi)$ 
here $c$ as well as $k$ are random nonzero constants.

Put on this wave transformation in (3.1), it can be rewritten as:

$R\left(u, u^{\prime}, u^{\prime \prime}, u^{\prime \prime \prime}, \ldots \quad \ldots \quad \ldots\right)=0$

where the superscripts require the ordinary derivative of $u$.

Phase 1: consider a formal solution of ODE in the subsequent structure

$u(\xi)=\sum_{i=0}^{n} a_{i} Y^{i}+\sum_{i=1}^{n} b_{i} Y^{-i}$

for which

$Y=\tanh (\mu \xi)$

where $\mu$ can be any arbitrary value.

Phase 2: Finding the homogeneous equilibrium among the highest order nonlinear terms and their derivatives in Eq. (3.3) determine the positive constant $\eta$.

Phase 3: By substituting solution (3.4) and (3.5) into Eq. (3.3) with the value of $\eta$ gotten in Phase 2, polynomials within $Y$ are obtained. Setting all of the coefficients of the resulting polynomials to zero yields a set of algebraic equations $a_{i}^{\prime} s$ along with $b_{i}^{\prime} s$. Solve these equations $a_{i}^{\prime} s$ along with $b_{i}^{\prime} s$ using symbolic computation tools like Maple.

Phase 4: By inserting the values from Phase 3 into Eq. (3.4) along with Eq. (3.5) and (3.1) we create closed feature moving wave solutions of the nonlinear evolution Eq. (3.4).

\section{Investigation of the Solutions}

In this segment, solitary wave explanations specifically the space time-fractional modified third-order $\mathrm{KdV}$ equation and the space time-fractional Telegraph equation by dint of the extended tanh-method designated in 'conformable fractional derivative. 


\subsection{The Space-Time Fractional Modified third order KdV Equation}

The space-time fractional modified third order $\mathrm{KdV}$ equation is

$D_{t}^{\alpha} u(x, t)+p u^{2}(x, t) D_{x}^{\beta} u(x, t)+q D_{x}^{3 \beta} u(x, t)=0.0<\alpha, \beta \leq 1$

where $p, q$ is a nonzero constant.

Let us consider the complex travelling waves transformation as

$\varsigma=\omega \frac{x^{\alpha}}{\alpha}-\lambda \frac{t^{\alpha}}{\alpha}, u(x, t)=u(\varsigma)$,

where $\omega, \lambda$ is the traveling wave's speed. The equation (4.1.1) is shortened to the following integer order ordinary differential equation (ODE) through the transformation (4.1.2):

$-\lambda u^{\prime}+\omega p u^{2} u^{\prime}+\omega^{3} q u^{\prime \prime \prime}=0$.

Integrating equation (4.1.3) with zero constant, we achieve

$3 \omega^{3} q u^{\prime \prime}-3 \lambda u+\omega p u^{3}=0$.

The balancing number is found 1 by balancing the highest order derivative term with the highest power nonlinear term. The equation (3.4) is then resolved as

$u(\varsigma)=a_{0}+a_{1} Y+b_{1} Y^{-1}$.

Take the place of (4.1.4) into (4.1.5) along with (3.5), in $Y$, the left side converts into a polynomial. When each of the polynomial's coefficients is set to zero, a set of algebraic equations emerges (intended used for plainness, we try to slip over them to exposition) for $a_{0}$, $a_{1}, b_{1}, \omega$ and $\lambda$.The subsequent outcomes are attained by put on computer algebra, such as Maple, to resolve this over determined series of equations:

\section{Case 1:}


$\omega=\frac{1}{6} \frac{\sqrt{-6 q} p}{q \mu} b_{1}, \lambda=\frac{\sqrt{-6 q} p}{18} \frac{b_{1}^{3}}{\mu}, a_{0}=0, a_{1}=0$ and $b_{1}=b_{1}$.

The principles of the constraints supplied into case 1 create explicit solution in terms of coth functions.

$u_{1}(x, t)=\operatorname{coth}\left(2 \sqrt{6} x^{1 / 4}-\frac{2 \sqrt{6} t^{1 / 4}}{9}\right)$.

We can rewrite this equation as follows:

$u_{2}(x, t)=\sqrt{1+\operatorname{cosech}\left(2 \sqrt{6} x^{1 / 4}-\frac{2 \sqrt{6} t^{1 / 4}}{9}\right)^{2}}$

\section{Case 2:}

$$
\omega=\frac{1}{6} \frac{\sqrt{-6 p q} p}{q \mu} a_{1}, \lambda=\frac{\sqrt{-6 p q} p}{18} \frac{b_{1}^{3}}{\mu}, a_{0}=0, a_{1}=a_{1} \text { and } b_{1}=0
$$

In terms of tanh functions, the ideals of the parameters stated in assortment 2 form an explicit result.

$u_{3}(x, t)=\tanh \left(\frac{4 x^{1 / 4}}{\sqrt{6}}-\frac{4 t^{1 / 4}}{3 \sqrt{6}}\right)$

This equation can be recreated using the following formula:

$u_{4}(x, t)=\sqrt{1-\operatorname{sech}\left(\frac{4 x^{1 / 4}}{\sqrt{6}}-\frac{4 t^{1 / 4}}{3 \sqrt{6}}\right)^{2}}$

\section{Case 3:}

$\omega=\frac{1}{6} \frac{\sqrt{-6 p q} p b_{1}}{q \mu}, \lambda=\frac{2}{9} \frac{\sqrt{-6 p q} p}{q \mu} b_{1}^{3}, a_{0}=0, a_{1}=b_{1}$ and $b_{1}=b_{1}$.

The principles of the parameters supplied in assortment 3 create an explicit solution in terms of the tanh and coth function.

$u_{5}(x, t)=\tanh \left(\frac{4 x^{1 / 4}}{\sqrt{6}}-\frac{8 t^{1 / 4}}{3 \sqrt{6}}\right)+\operatorname{coth}\left(\frac{4 x^{1 / 4}}{\sqrt{6}}-\frac{8 t^{1 / 4}}{3 \sqrt{6}}\right)$. 
The following formula can be used to reproduce this equation.

$u_{6}(x, t)=\sqrt{1-\operatorname{sech}\left(\frac{4 x^{1 / 4}}{\sqrt{6}}-\frac{8 t^{1 / 4}}{3 \sqrt{6}}\right)^{2}}+\sqrt{1+\operatorname{cosech}\left(\frac{4 x^{1 / 4}}{\sqrt{6}}-\frac{8 t^{1 / 4}}{3 \sqrt{6}}\right)^{2}}$

\section{Case 4:}

$\omega=\frac{1}{6} \frac{\sqrt{-6 p q} b_{1}}{q \mu}, \lambda=\frac{1}{9} \frac{\sqrt{-6 p q} p}{q \mu} b_{1}^{3}, a_{0}=0, a_{1=}-b_{1}$ and $b_{1}=b_{1}$.

In terms of the tanh and coth functions, the values of the parameters in assortment 4 establish an explicit solution.

$u_{7}(x, t)=-\tanh \left(\frac{4 x^{1 / 4}}{\sqrt{6}}-\frac{4 t^{1 / 4}}{9}\right)+\operatorname{coth}\left(\frac{4 x^{1 / 4}}{\sqrt{6}}-\frac{4 t^{1 / 4}}{9}\right)$.

The following formula can be used to reproduce this equation.

$u_{8}(x, t)=-\sqrt{1-\operatorname{sech}\left(\frac{4 x^{1 / 4}}{\sqrt{6}}-\frac{4 t^{1 / 4}}{9}\right)^{2}}+\sqrt{1+\operatorname{cosech}\left(\frac{4 x^{1 / 4}}{\sqrt{6}}-\frac{4 t^{1 / 4}}{9}\right)^{2}}$.

It is striking to note that the traveling wave solutions $u_{1}-u_{8}$ to the space-time fractional modified third-order KdV equation are all novel also more general. These solutions recite the circulation process of surface water waves and the electric circuit and multi constituent plasms in fluid mechanics.

\subsection{The space time fractional Telegraph equation}

The suggested approach uses in this subsection to observe more universal and novel closedform wave solutions to the space-time fractional Telegraph equation. The space-time fractional Telegraph equation is given by

$D_{t t}^{2 \alpha} u(x, t)-D_{x x}^{2 \alpha} u(x, t)+D_{t}^{\alpha} u(x, t)+\gamma u(x, t)+\beta u^{3}(x, t)=0$.

where $\alpha$ is a parameter recitation the order of the fractional space and time derivative. When $\alpha=-1$ equation (4.2.1) is termed the nonlinear Telegraph equation. Exploitation the fractional complex transform, 
$\epsilon=k \frac{x^{\alpha}}{\alpha}-c \frac{t^{\alpha}}{\alpha}, u(x, t)=u(\epsilon)$

where $c$ and $k$ be the constants. The equation (4.2.1) is diminished to the following integer order ordinary differential equation (ODE) through the transformation (4.2.2) and integrating equation with zero constant, we accomplish

$\left(c^{2}-k^{2}\right) u^{\prime \prime}-c u^{\prime}+\gamma u+\beta u^{3}=0$.

Balancing the highest order derivative term with the highest power nonlinear term the balancing number is found by 1 . The equation (3.4) is then resolved as

$u(\epsilon)=a_{0}+a_{1} Y+b_{1} Y^{-1}$.

Take the place of (4.2.3) into (4.2.4) along with (3.5), in $Y$, the left side converts into a polynomial. When each of the polynomial's coefficients is set to zero, a set of algebraic equations emerges (intended used for plainness, we try to slip over them to exposition) for $a_{0}$, $a_{1}, b_{1}, k$ and $c$.The subsequent outcomes are attained by put on computer algebra, such as Maple, to resolve this over determined series of equations:

\section{Family 1:}

$k=\frac{1}{2} \frac{I \sqrt{2 \gamma}}{\mu}, c=0, a_{0}=0, a_{1}=0$ and $b_{1}=\sqrt{-\frac{\gamma}{\beta}}$.

The parameters complete in Family 1 generates an explicit solution in terms of tanh functions.

$u_{9}(x, t)=\sqrt{-1} \operatorname{coth}\left(8 \sqrt{2} x^{1 / 4}\right)$.

Rewrite this equation as follows:

$u_{10}(x, t)=\sqrt{-1} \sqrt{1+\operatorname{cosech}\left(8 \sqrt{2} x^{1 / 4}\right)^{2}}$.

\section{Family 2:}

$k=\frac{1}{2} \frac{I \sqrt{2 \gamma}}{\mu}, c=0, a_{0}=0, a_{1}=\sqrt{-\frac{\gamma}{\beta}}$ and $b_{1}=0$. 
In terms of tanh functions, the ideals of the parameters stated within family 2 form a plain solution.

$u_{11}(x, t)=\sqrt{-1} \tanh \left(x^{1 / 4} / \sqrt{2}\right)$.

This equation can be recreated using the following formula:

$u_{12}(x, t)=\sqrt{1-\operatorname{sech}\left(x^{1 / 4} / \sqrt{2}\right)^{2}}$.

\section{Family 3:}

$k=\frac{1}{4} \frac{I \sqrt{2 \gamma}}{\mu}, c=0, a_{0}=0, a_{1}=\sqrt{-\frac{\gamma}{4 \beta}}$ and $b_{1}=\frac{1}{4} \frac{\gamma}{\sqrt{-\frac{\gamma}{4 \beta}}}$.

The parameters complete in Family 3 generates an explicit solution in terms of the tanh and coth function.

$u_{13}(x, t)=\frac{1}{2} \tanh \left(\sqrt{2} x^{1 / 4}\right)+\frac{1}{2} \operatorname{coth}\left(\sqrt{2} x^{1 / 4}\right)$.

\section{Family 4:}

$k=\frac{1}{4} \frac{I \sqrt{-2 \gamma+9 \gamma^{2}}}{\mu}, c=\frac{3 \gamma}{4 \mu}, a_{0}=\sqrt{-\frac{\gamma}{4 \beta}}, a_{1}=0$ and $b_{1}=\sqrt{-\frac{\gamma}{4 \beta}}$.

In terms of tanh and coth functions, the values of the parameters in assortment 4 create an explicit result.

$u_{14}(x, t)=\frac{1}{2}+\frac{1}{2} \operatorname{coth}\left(\sqrt{7} x^{1 / 4}-3 t^{1 / 4}\right)$

The following formula can be used to reproduce this equation.

$u_{15}(x, t)=\frac{1}{2}+\frac{1}{2} \sqrt{1+\operatorname{cosech}\left(\sqrt{7} x^{1 / 4}-3 t^{1 / 4}\right)^{2}}$. 


\section{Family 5:}

$k=\frac{1}{4} \frac{I \sqrt{-2 \gamma+9 \gamma^{2}}}{\mu}, c=-\frac{3 \gamma}{4 \mu}, a_{0}=\frac{1}{4} \frac{\gamma}{\sqrt{-\frac{\gamma}{4 \beta}}}, a_{1}=\sqrt{-\frac{\gamma}{4 \beta}}$ and $b_{1}=0$.

In terms of tanh and coth functions, the values of the parameters within family 4 establish an explicit answer.

$u_{16}(x, t)=\frac{1}{2}+\frac{1}{2} \tanh \left(\sqrt{7} x^{1 / 4}-3 t^{1 / 4}\right)$.

Correspondingly

$$
u_{17}(x, t)=\frac{1}{2}+\frac{1}{2} \sqrt{1-\operatorname{sech}\left(\sqrt{7} x^{1 / 4}-3 t^{1 / 4}\right]^{2}} .
$$

\section{Family 6:}

$k=\frac{1}{8} \frac{I \sqrt{-2 \gamma+9 \gamma^{2}}}{\mu}, c=-\frac{3 \gamma}{8 \mu}, a_{0}=\frac{1}{8 \beta} \frac{\gamma}{\sqrt{-\frac{\gamma}{16 \beta}}}, a_{1}=\sqrt{-\frac{\gamma}{16 \beta}}$ and $b_{1}=\frac{1}{16 \beta} \frac{\gamma}{\sqrt{-\frac{\gamma}{16 \beta}}}$.

The values of the parameters supplied in family 6 create an explicit solution in terms of the tanh and coth function.

$u_{18}(x, t)=\frac{1}{2}+\frac{1}{4} \tanh \left(\frac{\sqrt{7 x^{1 / 4}}}{2}+\frac{3 t^{1 / 4}}{2}\right)+\frac{1}{4} \operatorname{coth}\left(\frac{\sqrt{7 x^{1 / 4}}}{2}+\frac{3 t^{1 / 4}}{2}\right)$.

The following formula can be used to reproduce this equation.

$u_{19}(x, t)=\frac{1}{2}+\frac{1}{4} \sqrt{1-\operatorname{sech}\left(\frac{\sqrt{7 x^{1 / 4}}}{2}+\frac{3 t^{1 / 4}}{2}\right)^{2}}+\frac{1}{4} \sqrt{1+\operatorname{cosech}\left(\frac{\sqrt{7 x^{1 / 4}}}{2}+\frac{3 t^{1 / 4}}{2}\right)^{2}}$.

The solutions (4.2.5)-(4.2.15) attains the mentioned equations are all new and more general and this solutions can be explain to pulsatory blood movement among arteries also a onedimensional haphazard movement of bugs towards an obstacle, communication lines of all frequencies together with direct current also high-frequency etc. 


\section{Physical Description and Classification are part of the Visual Explanation.}

The physical explanation of the established traveling waves solutions to the space-time fractional modified third-order $\mathrm{KdV}$ equation and the space-time fractional Telegraph equations will be discussed in this section. The collected traveling-wave solutions of those equations are discussed in the three-dimensional plotline, the plot of contour, and plot of vector which are designed via Mathematica. Using those three sorts of pictorial descriptions, we may more explicitly characterize the physical sketch.

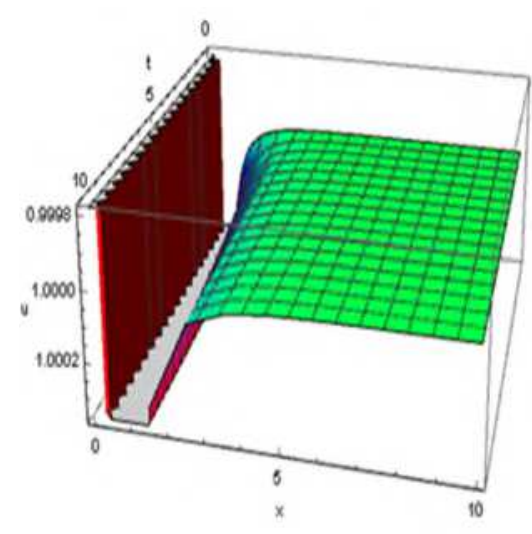

(a) Three-dimensional graph.

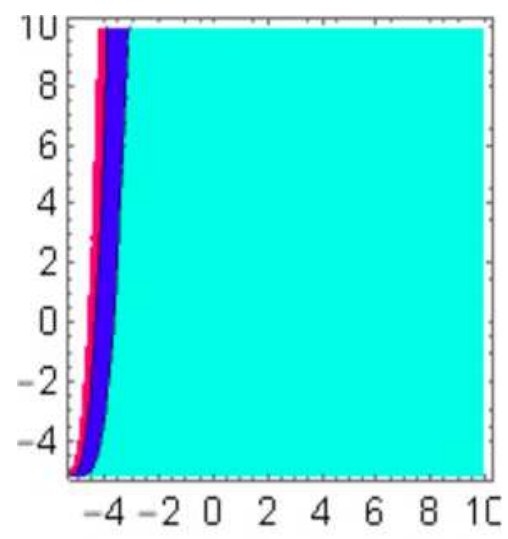

(b) Plot of contour.

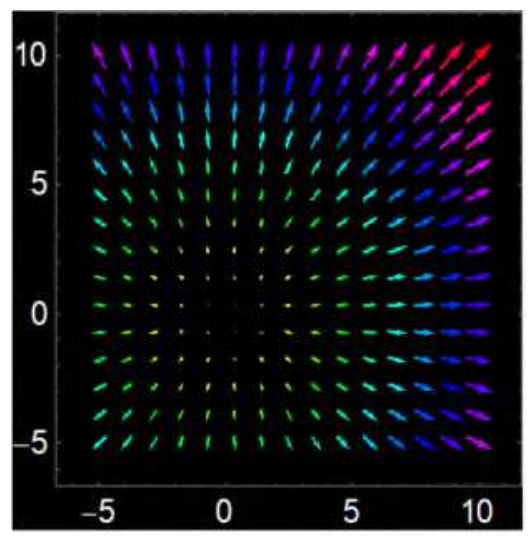

(c) Plot of vector.

Figure 01: Diagram of the periodic kink shape solution (4.1.6), representing (a) the threedimensional graph. (b) Plot of contour (c) and plot of vector of $\mathrm{u}_{1}(\mathrm{x}, \mathrm{t})$.

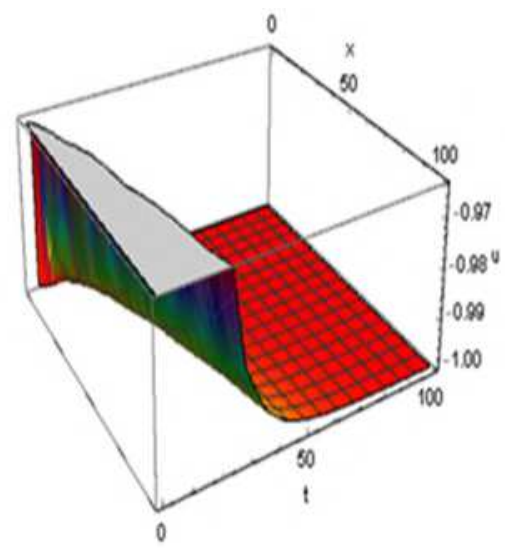

(a) Three-dimensional graph.

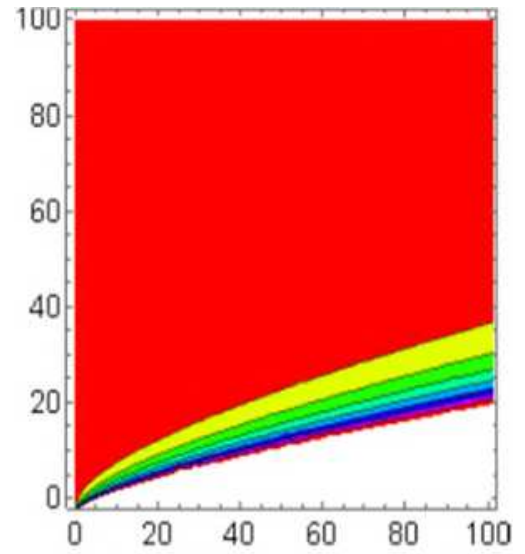

(b) Plot of contour.

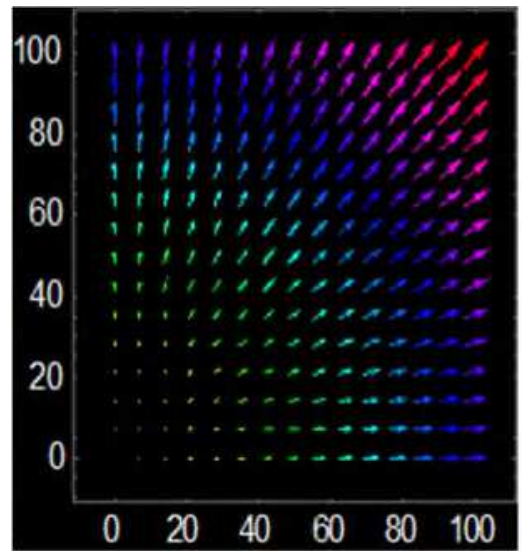

(c) Plot of vector.

Figure 02: Diagram of the kink shape wave solution (4.1.8), representing (a) the three-dimensional plotline (b) plot of contour (c) and plot of vector of $u_{3}(x, t)$. 


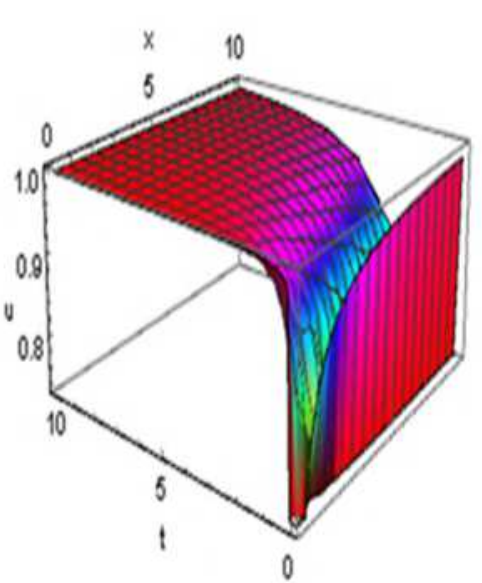

(a) Three-dimensional

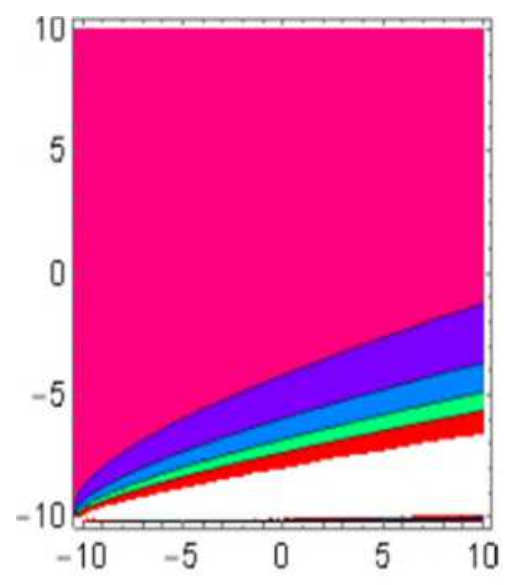

(b) Plot of contour.

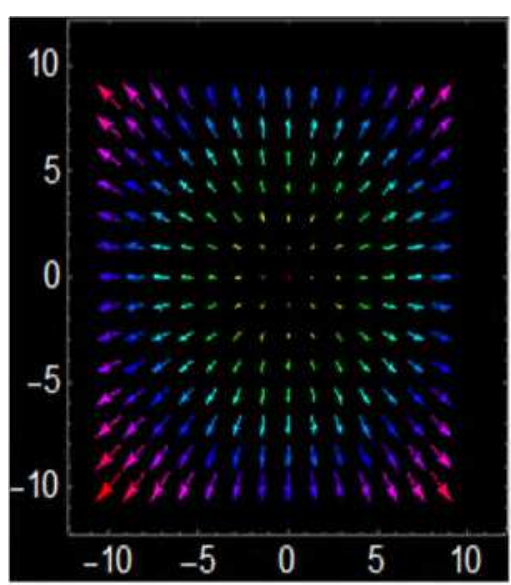

(c) Plot of vector. graph.

Figure 03: Diagram of the bell shape king wave solution (4.1.9), representing (a) the threedimensional plotline (b) plot of contour (c) and plot of vector of $u_{4}(x, t)$.

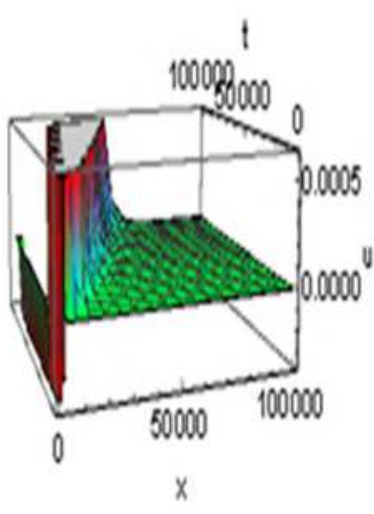

(a) Three-dimensional graph.

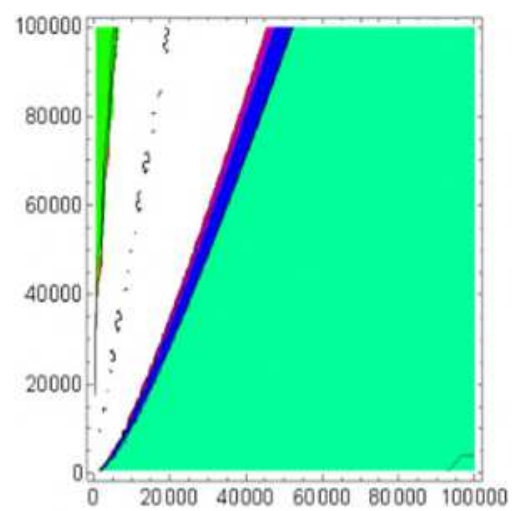

(b) Plot of contour.

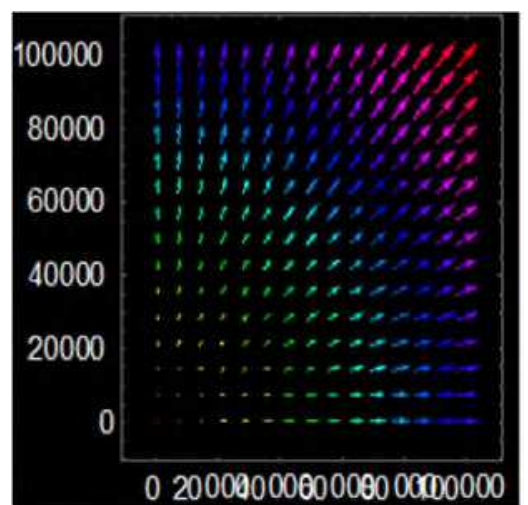

(c) Plot of vector.

Figure 04: Diagram of the soliton king shape wave solution (4.1.12), representing (a) the three-dimensional plotline (b) plot of contour (c) and plot of vector of $u_{7}(x, t)$.

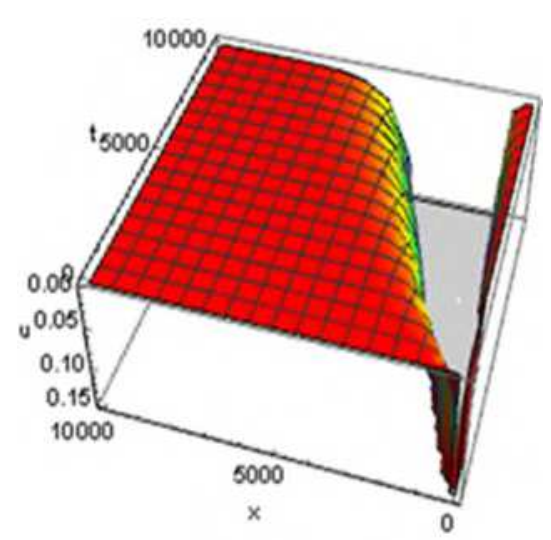

(a) Three-dimensional

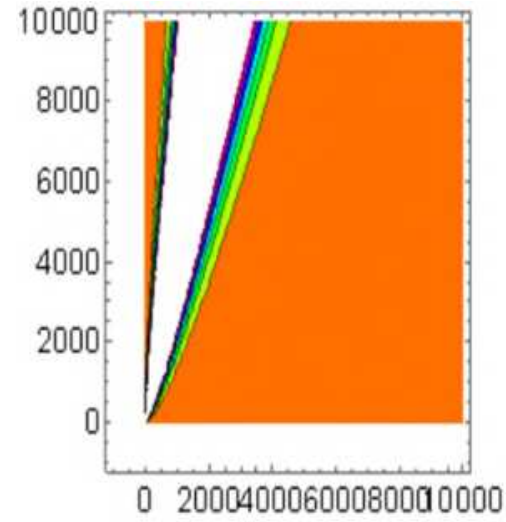

(b) Plot of contour.

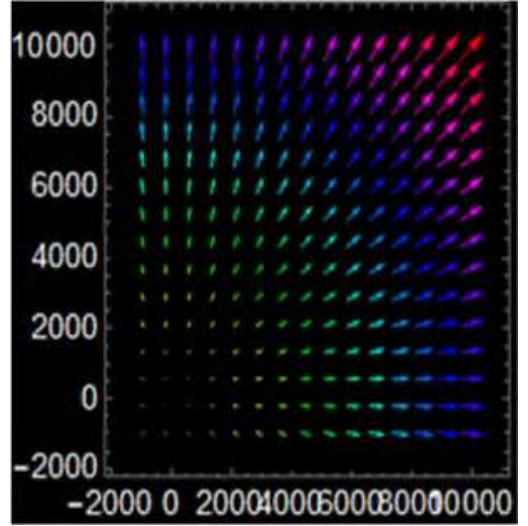

(c) Plot of vector. graph.

Figure 05: Illustration of the anti-bell king shape wave solution (4.1.13), representing (a) the threedimensional plotline (b) plot of contour (c) and plot of vector of $\mathrm{u}_{8}(x, t)$. 


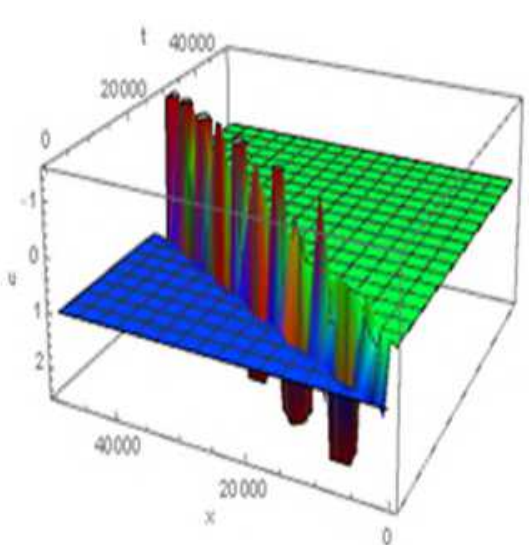

(a) Three-dimensional graph.

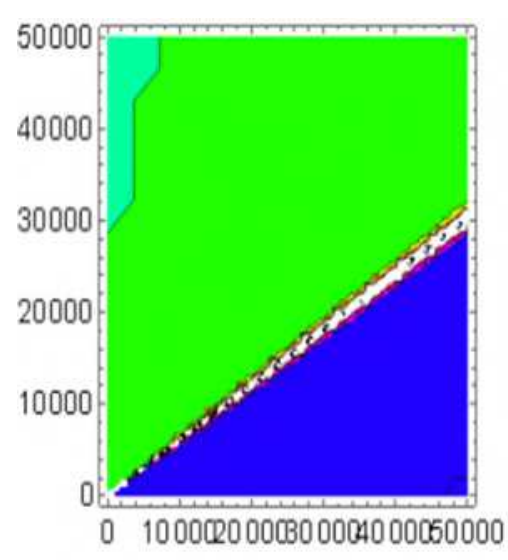

(b) Plot of contour.

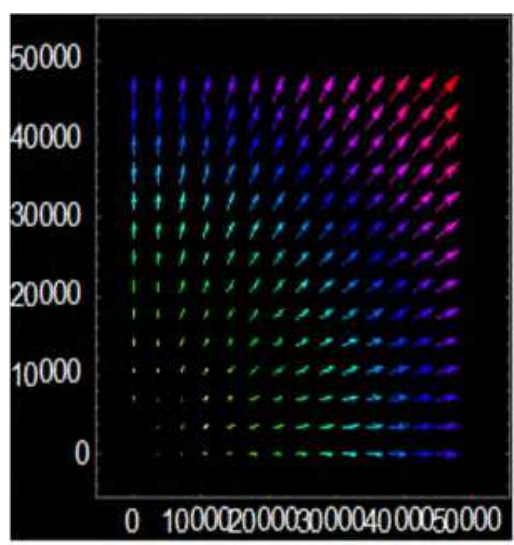

(c) Plot of vector.

Figure 06: Illustration of the multiple soliton shape (solutions which behave at large time as a sum of solitons) wave solution (4.2.10), representing (a) the three-dimensional plotline (b) plot of contour (c) and plot of vector of $\mathrm{u}_{14}(x, t)$.

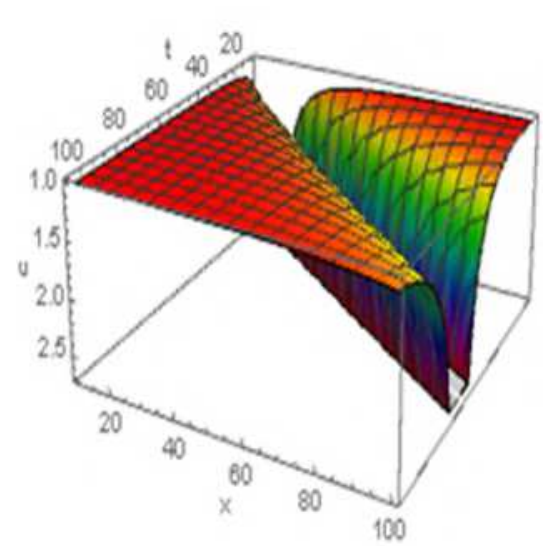

(a) Three-dimensional

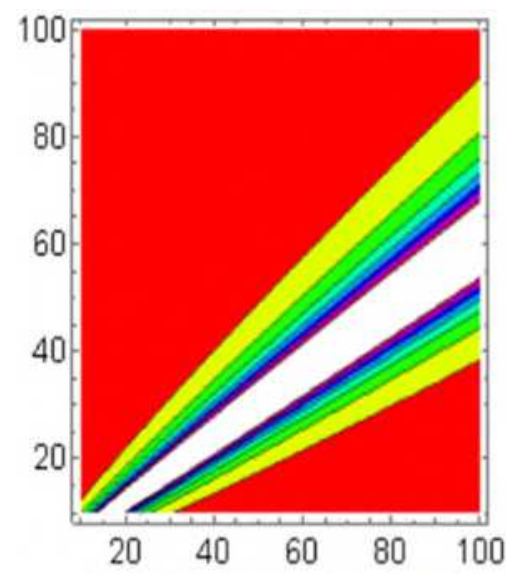

(b) Plot of contour.

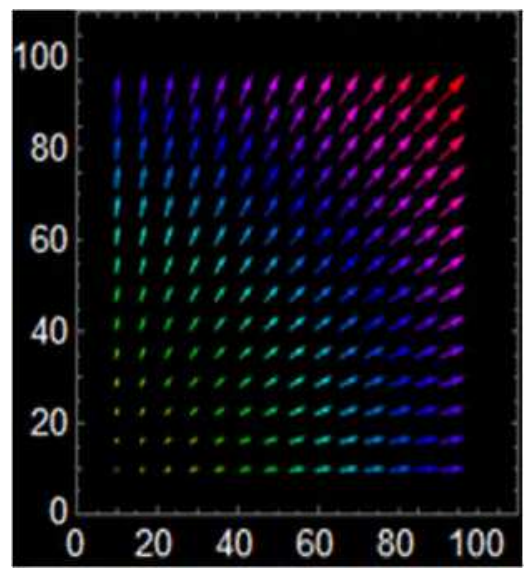

(c) Plot of vector. graph.

Figure 07: Illustration of the singular bell shape wave solution (4.2.11), representing (a) the three-dimensional plotline (b) plot of contour (c) and plot of vector of $\mathrm{u}_{15}(x, t)$.

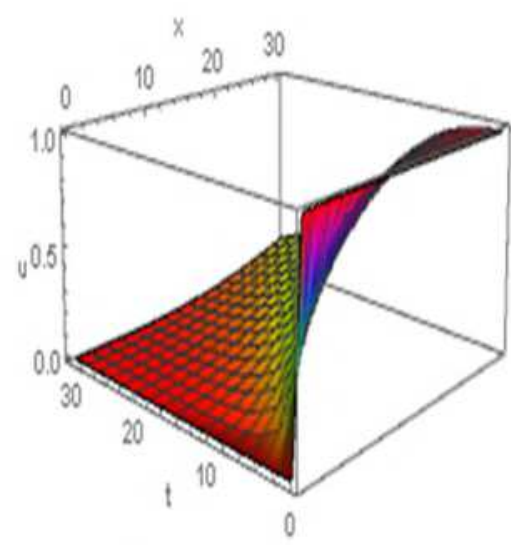

(a) Three-dimensional graph.

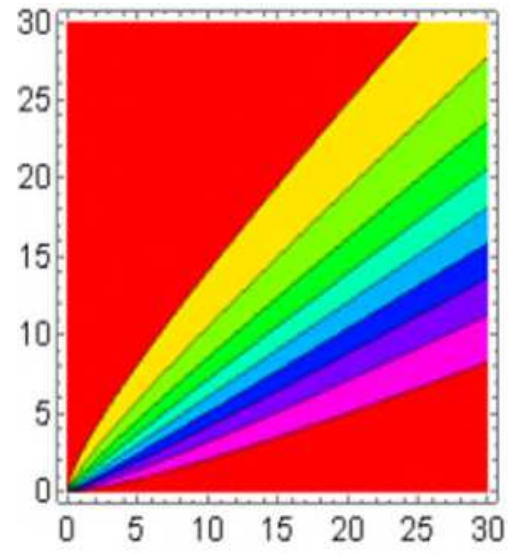

(b) Plot of contour.

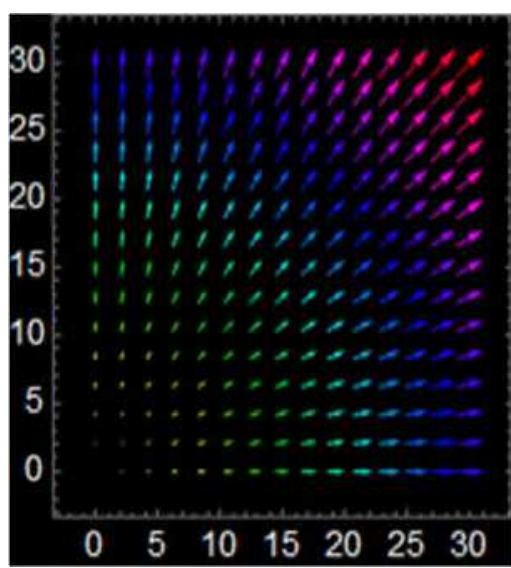

(c) Plot of vector.

Figure 08: Illustration of the single soliton shape wave solution (4.2.12), representing (a) the three-dimensional plotline (b) plot of contour (c) and plot of vector of $\mathrm{u}_{16}(x, t)$. 


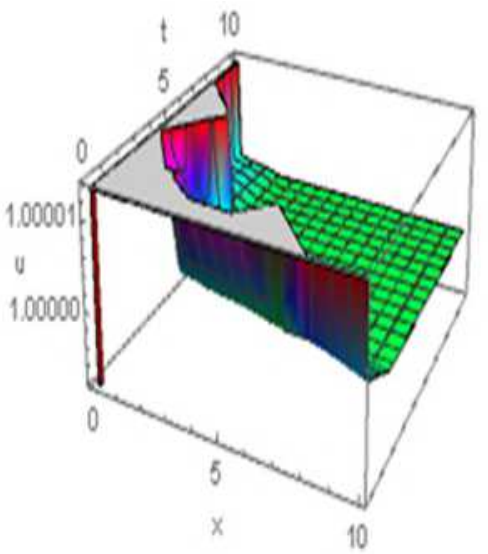

(a) Three-dimensional graph.

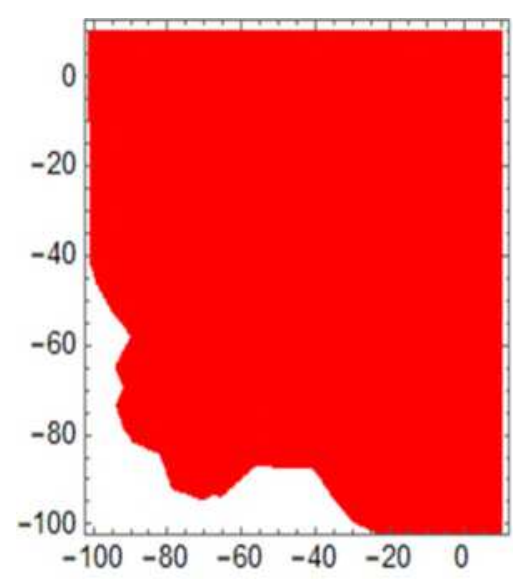

(b) Plot of contour.

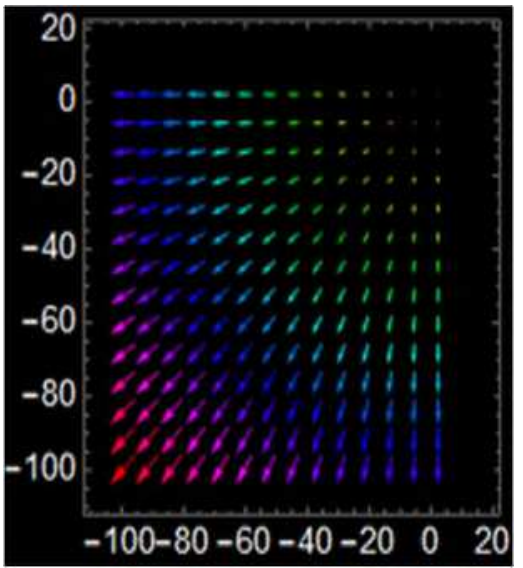

(c) Plot of vector.

Figure 09: Diagram of the single soliton shape wave solution (4.2.15), representing (a) the threedimensional plotline (b) plot of contour (c) and plot of vector of $\mathrm{u}_{19}(x, t)$.

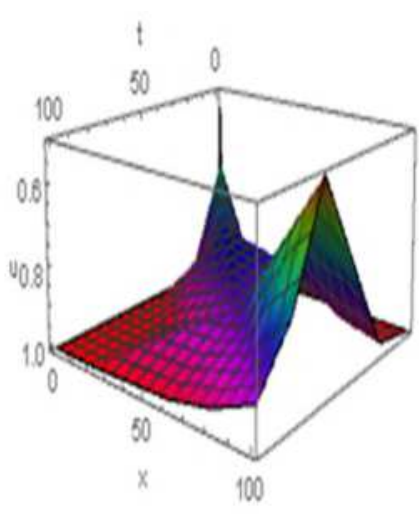

(a) Three-dimensional graph.

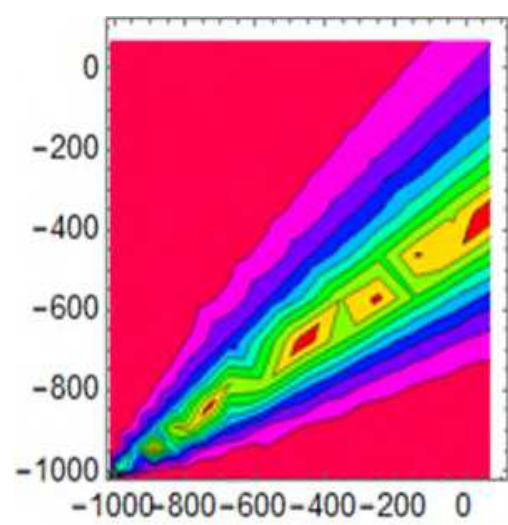

SSS

(b) Plot of contour.

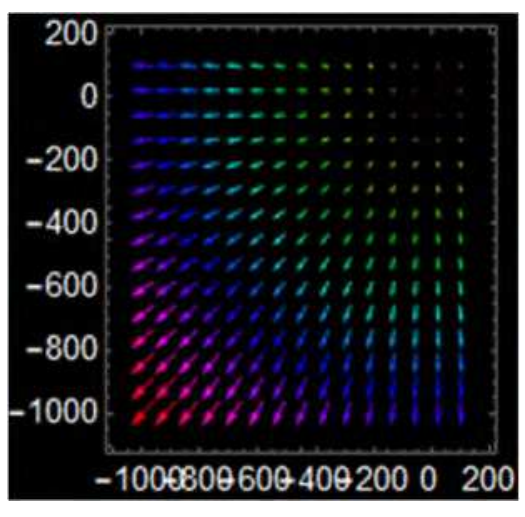

(c) Plot of vector.

Figure 10: Diagram of the double solitons shape wave solution (4.2.13), representing (a) the three-dimensional plotline (b) plot of contour (c) and plot of vector of $\mathrm{u}_{17}(x, t)$.

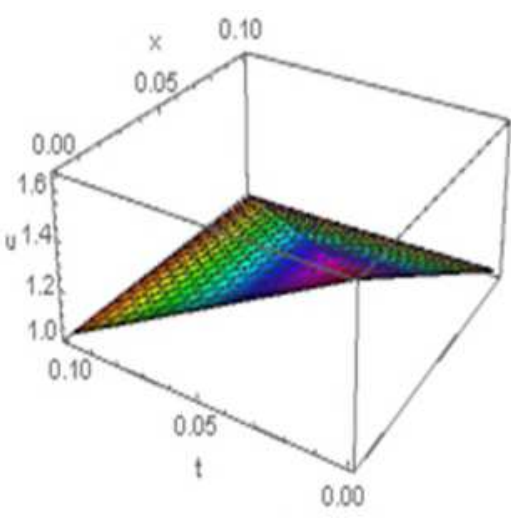

(a) Three-dimensional graph.

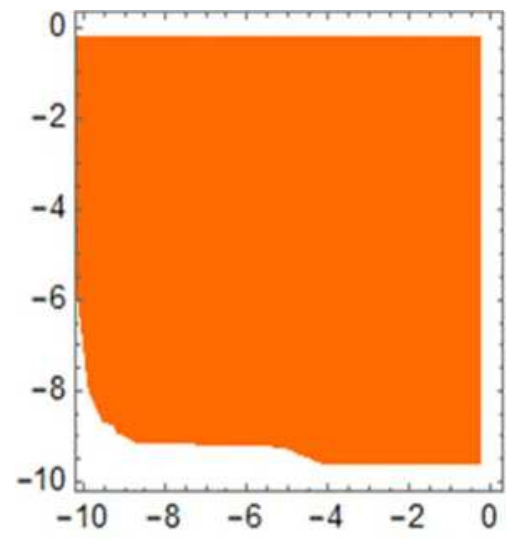

(b) Plot of contour.

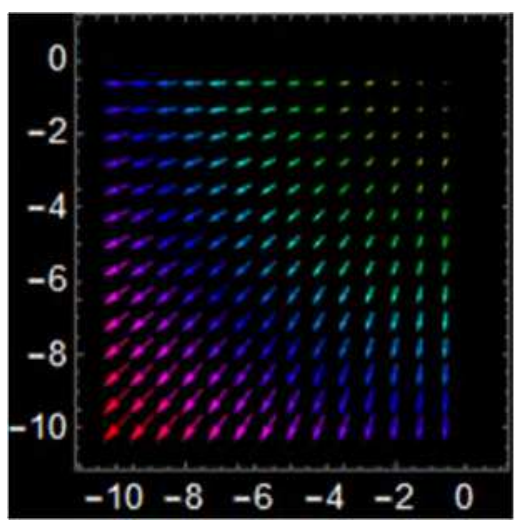

(c) Plot of vector.

Figure 11: Diagram of the compaction wave solution (4.2.14), representing (a) the threedimensional plotline (b) plot of contour (c) and plot of vector of $\mathrm{u}_{18}(x, t)$. 


\section{Physical clarification of the resolution}

In this segment graphic delegation also physical amplification of the resolution of the derived solutions of nonlinear fractional differential equations through the time fractional modified third-order KdV equation and Telegraph equation are recapitulated. Solution $u_{1}(x, t)$ in fig-1 for space-time fractional modified third-order $\mathrm{KdV}$ equation illustrates periodic kink shape wave solution, within the interval $-5<x<10$ and $-5<t<10$ and with the values $\mu=$ $1, p=1, q=-1, \beta=\frac{1}{4}, \alpha=\frac{1}{4}$. Periodic king wave is a recapping nonstop pattern which regulates its wavelength and frequency. similarly, solution $u_{2}(x, t)$ and $u_{5}(x, t)$ delivers the same types of solutions like periodic king wave for the values of same intervals but for the simplicity those are omitted here. Solution $u_{3}(x, t)$ in fig- 2 for space-time fractional modified third-order $\mathrm{KdV}$ equation illustrates the kink shape wave solution with the interval $0<x<$ 100 and $0<t<100$ for the values $\mu=1, p=1, q=-1, \beta=\frac{1}{4}, \alpha=\frac{1}{4}$. Kink shape wave solution be a wave which travels from one asymptotic spot to additional asymptotic spot wave. Similarly, for space-time fractional Telegraph equation, solutions $u_{6}(x, t), u_{9}(x, t), u_{10}(x, t)$, $u_{11}(x, t), u_{12}(x, t)$ for the values $\mu=1, p=1, q=-1, \beta=\frac{1}{4}, \alpha=\frac{1}{4}$, all are represent the kink wave solution, which are hide here. In fig-3 solution $\mathrm{u}_{4}(\mathrm{x}, \mathrm{t})$ for space-time fractional modified third-order $\mathrm{KdV}$ equation shows bell shape wave solution through the interval $-10<$ $x<10$ and $-10<t<10$ for the values $\mu=1, p=1, q=-1, \beta=\frac{1}{4}, \alpha=\frac{1}{4}$. Bell shape wave is a continuous circulation through no breaks among principles. Fig-4 is the diagram of the soliton king shape of $\mathrm{u}_{7}(\mathrm{x}, \mathrm{t})$ for the values $\mu=1, p=1, q=-1, \beta=\frac{1}{4}, \alpha=\frac{1}{4}$ with the interval $500<x<100000$ and $500<t<100000$. Soliton king shape is a confined disorder which circulates alike a wave. Solution $\mathrm{u}_{8}(x, t)$ for space-time fractional modified 
third-order $\mathrm{KdV}$ equation shows anti-bell king wave solution for the values $\mu=1, p=1, q=$ $-1, \beta=\frac{1}{4}, \alpha=\frac{1}{4}$ with the interval $-1000<x<10000$ and $-1000<t<10000$ in fig-5.

The solution of $\mathrm{u}_{14}(x, t)$ imply the type of multiple soliton shape solution, intended for the standards $\mu=1, I=1, \gamma=-1, \beta=\frac{1}{4}, \alpha=\frac{1}{4} \quad$ and $0<x<50000,0<t<50000$ is symbolized by Fig.-6 which are represented space-time fractional Telegraph equation. The solution of $\mathrm{u}_{15}(x, t)$ imply the type of singular bell shape wave solution, intended for the standards $\quad \mu=1, I=1, \gamma=-1, \beta=\frac{1}{4}, \alpha=\frac{1}{4} \quad$ and $\quad 10<x<100, \quad 10<t<100$ is symbolized by Fig.-7. For the case of space-time fractional Telegraph equation, fig- 8 demonstrates single solitons shape wave solution for the solution of $\mathrm{u}_{16}(x, t)$ considering the values of $\mu=1, I=1, \gamma=-1, \beta=\frac{1}{4}, \alpha=\frac{1}{4}$ with the interval $0<x<30,0<t<30$, and also fig- 9 demonstrates single solitons shape wave solution for the solution of $\mathrm{u}_{19}(x, t)$ considering the values of $\mu=1, I=1, \gamma=-1, \beta=\frac{1}{4}, \alpha=\frac{1}{4}$ with the interval $-100<x<$ $10,-100<t<10$. Singular solitons are a form of solitary wave that has a singularity, which is typically an infinite discontinuity. When the center point of the solitary wave is imaginary, singular solitons can be bound to it. In fig-10, the double solitons shape wave solution is depicted, for the solution of $\mathrm{u}_{17}(x, t)$ (space-time fractional Telegraph equation) with the values of $\mu=1, I=1, \gamma=-1, \beta=\frac{1}{4}, \alpha=\frac{1}{4}$ within the interval $100<x<-1000$ and $100<t<-1000$. Double solitons is known as a bion, or in systems where the bound state periodically oscillates, a breather. Solution $\mathrm{u}_{18}(x, t)$, for space-time fractional Telegraph equation illustrates compaction wave solution in fig-11, for the values of $\mu=1, I=1, \gamma=$ $-1, \beta=\frac{1}{4}, \alpha=\frac{1}{4}$ with the interval $-10<x<0.1$ and $-10<t<0.1$. Compaction wave solution defined as a propagating disturbance of the solid volume fraction of the granular material. 


\section{Conclusion}

In summary, this manuscript has built scores of innovative, extra universal, and wide-ranging solitary wave explanations employing two dependable ways relating conformable fractional derivative, in addition, extended tanh-method to the nonlinear space time-fractional modified third-order Kdv equation and the nonlinear space time-fractional Telegraph equation. The obtained results illustrate that both equations accept a large number of closed-form investigative explanations with arbitrarily conversant irreversible parameters. The recognized outcomes reveal amusing dynamical constructions of soliton results in the categories of single solitons, double solitons, bell types waves, kink-type waves, imaginary waves also multiple solitons. The dynamics of solitary waves have been realistically described within relations of space and time coordinates, revealing the reliability of the methodologies employed. The accurateness of the data produced in these learning was confirmed by means of the computational software Maple by re-entering them into NLFPDEs and confirming that were correct. These soliton solutions will be beneficial in soliton theory, nonlinear wave physics, plasma physics, optical engineering, oceanography, fluid dynamics, and engineering physics. It is remarkable to the indication that the extended tanh-function scheme formed an extensive assortment of computational wave solutions to the main equation. The existing technique's foremost benefit is that it generates more general solutions with arbitrary known-constant constraints that also can be exerted to several categories of NLFPDEs.

\section{Conflict of interest:}

We guarantee that in this article none of the authors have any contest of interests. 


\section{Author's contribution}

Ummay Sadia: Software, Data Curation, Writing, Formal Analysis.

Mohammad Asif Arefin: Software, Data Curation, Writing, Investigation.

Mustafa Inc: Investigation, Validation.

M. Hafiz Uddin: Conceptualization, Supervision, Writing-Reviewing Editing, Validation.

\section{Data Availability Statements}

Data sharing not applicable to this article as no datasets were generated or analyzed during the current study.

\section{References}

[1] Du M, Wang Z, Hu H. Measuring memory with the order of fractional derivative. Sci Rep 2013;3:1-3. https://doi.org/10.1038/srep03431.

[2] Caputo M, Fabrizio M. A new definition of fractional derivative without singular kernel. Prog Fract Differ Appl 2015;1:73-85. https://doi.org/10.12785/pfda/010201.

[3] Zheng B. Exp-function method for solving fractional partial differential equations. Sci World J 2013;2013. https://doi.org/10.1155/2013/465723.

[4] Čermák J, Kisela T. Stability properties of two-term fractional differential equations. Nonlinear Dyn 2015;80:1673-84. https://doi.org/10.1007/s11071-014-1426-x.

[5] Alzai JF. The Fractional Sub-Equation Method and Exact Analytical Solutions for Some Nonlinear Fractional PDEs. Am J Math Anal 2013;1:14-9. https://doi.org/10.12691/ajma-1-1-3.

[6] Meng F, Feng Q. A new fractional subequation method and its applications for spacetime fractional partial differential equations. J Appl Math 2013;2013. https://doi.org/10.1155/2013/481729. 
[7] Guo S, Mei L, Li Y, Sun Y. The improved fractional sub-equation method and its applications to the space-time fractional differential equations in fluid mechanics. Phys Lett Sect A Gen At Solid State Phys 2012;376:407-11. https://doi.org/10.1016/j.physleta.2011.10.056.

[8] Feng Z. The first-integral method to study the Burgers - Korteweg - de Vries equation n.d.;343.

[9] Younis M. The First Integral Method for Time-Space Fractional Differential Equations. J Adv Phys 2013;2:220-3. https://doi.org/10.1166/jap.2013.1074.

[10] Meng F. A new approach for solving fractional partial differential equations. J Appl Math 2013;2013. https://doi.org/10.1155/2013/256823.

[11] Li C, Chen A, Ye J. Numerical approaches to fractional calculus and fractional ordinary differential equation. J Comput Phys 2011;230:3352-68. https://doi.org/10.1016/j.jcp.2011.01.030.

[12] Varieschi GU. Applications of Fractional Calculus to Newtonian Mechanics. J Appl Math Phys 2018;06:1247-57. https://doi.org/10.4236/jamp.2018.66105.

[13] Cui M. Compact finite difference method for the fractional diffusion equation. $\mathrm{J}$ Comput Phys 2009;228:7792-804. https://doi.org/10.1016/j.jcp.2009.07.021.

[14] Huang Q, Huang G, Zhan H. A finite element solution for the fractional advectiondispersion equation. Adv Water Resour 2008;31:1578-89. https://doi.org/10.1016/j.advwatres.2008.07.002.

[15] El-Sayed AMA, Gaber M. The Adomian decomposition method for solving partial differential equations of fractal order in finite domains. Phys Lett Sect A Gen At Solid State Phys 2006;359:175-82. https://doi.org/10.1016/j.physleta.2006.06.024. 
[16] El-Sayed AMA, Behiry SH, Raslan WE. Adomian's decomposition method for solving an intermediate fractional advection-dispersion equation. Comput Math with Appl 2010;59:1759-65. https://doi.org/10.1016/j.camwa.2009.08.065.

[17] Odibat Z, Momani S. A generalized differential transform method for linear partial differential equations of fractional order. Appl Math Lett 2008;21:194-9. https://doi.org/10.1016/j.aml.2007.02.022.

[18] He J. Variational iteration method for delay differential equations. Commun Nonlinear Sci Numer Simul 1997;2:235-6. https://doi.org/10.1016/S1007-5704(97)90008-3.

[19] Wu G cheng, Lee EWM. Fractional variational iteration method and its application. Phys Lett Sect A Gen At Solid State Phys 2010;374:2506-9. https://doi.org/10.1016/j.physleta.2010.04.034.

[20] Guo S, Mei L. The fractional variational iteration method using He's polynomials. Phys Lett Sect A Gen At Solid State Phys 2011;375:309-13. https://doi.org/10.1016/j.physleta.2010.11.047.

[21] Jafari H, Seifi S. Homotopy analysis method for solving linear and nonlinear fractional diffusion-wave equation. Commun Nonlinear Sci Numer Simul 2009;14:2006-12. https://doi.org/10.1016/j.cnsns.2008.05.008.

[22] He JH. Coupling method of a homotopy technique and a perturbation technique for non-linear problems. Int J Non Linear Mech 2000;35:37-43. https://doi.org/10.1016/S0020-7462(98)00085-7.

[23] Khatun MA, Arefin MA, Uddin MH. Abundant Explicit Solutions to Fractional Order Nonlinear Evolution Equations 2021;2021.

[24] Uddin MH, Arefin MA, Akbar MA. New Explicit Solutions to the Fractional-Order Burgers ' Equation 2021;2021. 
[25] Uddin MH, Khatun MA, Arefin MA, Akbar MA. Abundant new exact solutions to the fractional nonlinear evolution equation via Riemann-Liouville derivative. Alexandria Eng J 2021;60:5183-91. https://doi.org/10.1016/j.aej.2021.04.060.

[26] Khatun MA, Asif M, Uddin MH, Baleanu D, Akbar MA. Results in Physics Explicit wave phenomena to the couple type fractional order nonlinear evolution equations. Results Phys 2021;28:104597. https://doi.org/10.1016/j.rinp.2021.104597.

[27] Raza N, Zubair A, Arshed S, Mahmoud EE. Optical solitons in birefringent fibers with quadratic-cubic nonlinearity using three integration architectures 2021;025121:1-16. https://doi.org/10.1063/5.0038038.

[28] Zhang J, Yang X. A class of efficient difference method for time fractional reactiondiffusion equation. Comput Appl Math 2018;37:4376-96. https://doi.org/10.1007/s40314-018-0579-5.

[29] Mophou GM. Existence and uniqueness of mild solutions to impulsive fractional differential equations. Nonlinear Anal Theory, Methods Appl 2010;72:1604-15. https://doi.org/10.1016/j.na.2009.08.046.

[30] Liu F, Anh V V., Turner I, Zhuang P. Time fractional advection-dispersion equation. J Appl Math Comput 2003;13:233-45. https://doi.org/10.1007/BF02936089.

[31] Jiang W, Lin Y. Approximate solution of the fractional advection-dispersion equation. Comput Phys Commun 2010;181:557-61. https://doi.org/10.1016/j.cpc.2009.11.004.

[32] Pandey RK, Singh OP, Baranwal VK. An analytic algorithm for the space-time fractional advection-dispersion equation. Comput Phys Commun 2011;182:1134-44. https://doi.org/10.1016/j.cpc.2011.01.015.

[33] Xue C, Nie J, Tan W. An exact solution of start-up flow for the fractional generalized Burgers' fluid in a porous half-space. Nonlinear Anal Theory, Methods Appl 
2008;69:2086-94. https://doi.org/10.1016/j.na.2007.07.047.

[34] Molliq R Y, Noorani MSM, Hashim I. Variational iteration method for fractional heatand wave-like equations. Nonlinear Anal Real World Appl 2009;10:1854-69. https://doi.org/10.1016/j.nonrwa.2008.02.026.

[35] Arshed S, Biswas A, Zhou Q, Khan S, Adesanya S. Optik Optical solitons pertutabation with Fokas-Lenells equation by $\exp (-\phi(\xi))$-expansion method. Opt Int J Light Electron Opt 2019;179:341-5. https://doi.org/10.1016/j.ijleo.2018.10.136.

[36] Simulations C. The mathematics of suspensions: kac walks and asymptotic analyticity 2000:39-50.

[37] Biazar J, Ebrahimi H, Ayati Z. An Approximation to the Solution of Telegraph Equation by Variational Iteration Method 2009. https://doi.org/10.1002/num.

[38] Yildırım A. He's homotopy perturbation method for solving the space- and timefractional telegraph equations 2010;7160. https://doi.org/10.1080/00207160902874653.

[39] Avazzadeh HHZ, Machado JAT. Numerical approach for solving variable - order space - time fractional telegraph equation using transcendental Bernstein series. Eng Comput 2019;0:0. https://doi.org/10.1007/s00366-019-00736-x.

[40] Al-shawba AA, Abdullah FA. expansion method Travelling Wave Solutions for Fractional Boussinesq Equation Using Modified ( G '/ G ) Expansion Method 2018;020036. https://doi.org/10.1063/1.5041567.

[41] Shah R, Farooq U, Khan H, Baleanu D, Kumam P. Fractional View Analysis of Third Order Kortewege-De Vries Equations , Using a New Analytical Technique 2020;7:111. https://doi.org/10.3389/fphy.2019.00244. 
[42] Sepehrian B, Shamohammadi Z. Arabian Journal of Mathematics A high order method for numerical solution of time-fractional $\mathrm{KdV}$ equation by radial basis functions. Arab J Math 2018;7:303-15. https://doi.org/10.1007/s40065-018-0197-5.

[43] Khalil R, Al Horani M, Yousef A, Sababheh M. A new definition of fractional derivative. J Comput Appl Math 2014;264:65-70.

https://doi.org/10.1016/j.cam.2014.01.002.

[44] Wazwaz AM. The extended tanh method for abundant solitary wave solutions of nonlinear wave equations. Appl Math Comput 2007;187:1131-42. https://doi.org/10.1016/j.amc.2006.09.013. 